\title{
VOR Times Series for an Aircraft Trajectory in the Presence of Wind Turbines
}

\author{
Seif Ben-Hassine \\ ENAC TELECOM EMA \\ Toulouse, France \\ seif.ben-hassine@enac.fr
}

\author{
Alexandre Chabory \\ ENAC TELECOM EMA \\ Toulouse, France \\ alexandre.chabory@enac.fr
}

\author{
Christophe Morlaas \\ ENAC TELECOM EMA \\ Toulouse, France \\ christophe.morlaas@enac.fr
}

\author{
Rémi Douvenot \\ ENAC TELECOM EMA \\ Toulouse, France \\ remi.douvenot@enac.fr
}

\begin{abstract}
In air navigation, multipath due to large ground scatterers such as wind turbines is a major source of bearing error for VOR systems. Existing models to assess this error neglect the rapid multipath change in time. In this article, this is discussed by computing time series for the VOR signal and multipath.
\end{abstract}

Index Terms-Propagation, multipath, wind turbines, VOR

\section{INTRODUCTION}

Wind turbines settled close to VHF Omnidirectional Range (VOR) beacons constitute a source of multipath that can yield unacceptable errors in the bearing estimated by aircraft receivers. In recent years, several models have been proposed to assess this error source [1]-[3]. For determining the multipath characteristics, these models generally rely on either one computational-electromagnetics method or on the hybridization of several methods, e.g. uniform theory of diffraction, the method of moments, or physical optics. For determining the bearing error from the multipath characteristics, the formulas proposed in [4] are generally used. In [5], [6], we have proposed such a model by hybridizing the parabolic equation method (PE) with physical optics (PO). The results have been compared with measurements from in-flight tests.

In the previous models, a limitation is that everything is assumed static. In reality, since the aircraft is moving, multipath changes rapidly in time. Thus, filters and response times of the receiver may render the bearing errors estimated by the formulas in [4] inaccurate.

In this article, we propose to generate VOR times series for the signal and multipath parameters along an aircraft trajectory. This allows to analyze the dynamics of the multipath.

In Section 2, the configuration is exposed. In Section 3, an overview of the electromagnetic model is presented and the time-series generation is detailed. In Section 4, simulation results for a complex scenario are shown in static configuration.

\section{Configuration}

As illustrated in Figure 1, the configuration contains the VOR beacon, the wind turbines, the terrain and the aircraft trajectory. The VOR beacon is characterized by its position, frequency, power and radiation pattern. For the wind turbines,

The authors thank ENAC and DGAC/DTI and région Occitanie for funding this work. a generic model with dielectric blades is used for which all the sizes and angles are tunable. The terrain is characterized by its relief and dielectric characteristics.

The aircraft trajectory is defined from a series of way-points at which the aircraft speed is known. Between way-points, the trajectory is assumed circular with a constant acceleration. Since our aim is to obtain time series, the trajectory is sampled using a constant time step. A particular attention must be devoted to the choice of this time step. Indeed, to capture completely the signal variations in space, two consecutive samples must be separated by a sufficiently short distance with respect to the wavelength.

\section{Multipath COMPUTATION}

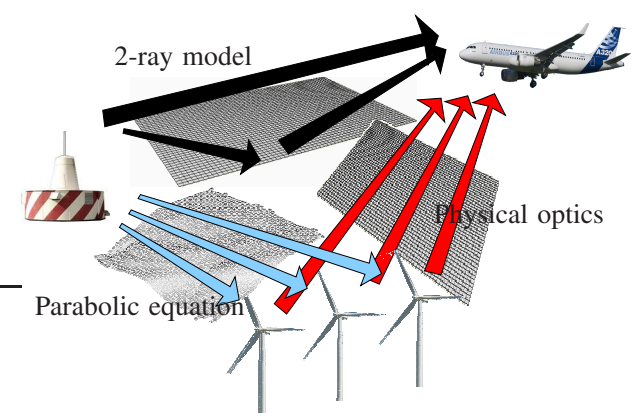

Fig. 1. Overview of the method to compute multipath.

At each point of the trajectory, multipath come from large scatterers on the ground. They are characterized by an amplitude, a phase and an azimuth. The latter corresponds to the azimuth of the scatterer with respect to the VOR beacon. At each trajectory point, we use the method proposed in [5] to compute the multipath parameters. This method relies on a hybridization of the parabolic equation method (PE) with physical optics (PO). Four steps are necessary. First, $\mathrm{PE}$ is used between the VOR beacon and the wind turbines taking into account the terrain characteristics. This provides an accurate illumination for the wind turbines. Second, from this illumination, the currents on the masts, boxes and blades are calculated upon the physical optics approximation. Third, the scattered fields are computed at the aircraft position. These fields are finally used to obtain the multipath characteristics at the output of the receiver antenna. 
This method gives time series for the multipath characteristics. It can also be used to assess the direct, scattered and total fields along the aircraft trajectory.

\section{Simulation Results}

We consider the configuration exposed in Figure 2. The VOR beacon works at $113.8 \mathrm{MHz}$ with a power of $25 \mathrm{~W}$. There are 4 wind turbines of type Enercon E82. The nearest windturbine is at $500 \mathrm{~m}$ from the beacon. The aircraft trajectory starts at $2.5 \mathrm{~km}$ from the beacon. It contains 5 way-points at which speeds are between $180 \mathrm{~km} / \mathrm{h}$ and $288 \mathrm{~km} / \mathrm{h}$. To have at least 5 points per wavelength, we choose a time step of 6.63 ms.

In Figure 3, time series of the electric field are shown. We observe that the field is significantly influenced by the wind turbines. In Figure 4, we display the power azimuth profile, i.e. the relative power and azimuth of the multipath at each epoch, where we distinguish the 4 multipath associated with the 4 wind turbines. Finally, the VOR error obtained from the formula in [4] for a static scenario is displayed in Figure 5. From 0 to $270 \mathrm{~s}$, and from $350 \mathrm{~s}$ to $680 \mathrm{~s}$, the VOR error is due to several multipaths, which explains the fast fluctuations. The maxima of the VOR error correspond to the maxima of the relative power of the multipaths for azimuth close to $90^{\circ}$. Between 270 and $350 \mathrm{~s}$, the multipath due to wind turbine 2 has no effect (the aircraft, the VOR station and the wind turbine are aligned). The power azimuth profile indicates that the multipath due to wind turbine 1 is dominating. Therefore, the slow variation is the scalloping effect corresponding to the presence of one signal scatterer.

\section{CONCLUSion}

In this article, the computation of time series for VOR signals in the presence of multipath due to wind turbines has been presented and illustrated in a complex scenario. For future works, these time series will be sent to a digital VOR receiver to assess the influence of the multipath changes in time on the bearing error. The Doppler effect due to blade rotation and receiver movement will be observed.

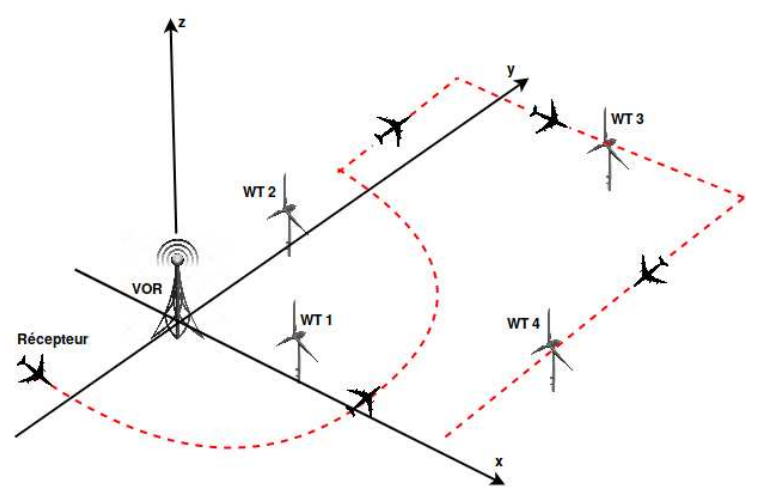

Fig. 2. Configuration.

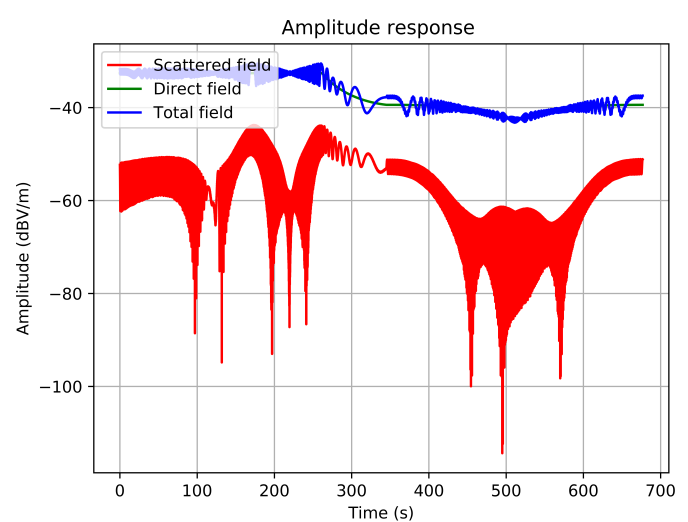

Fig. 3. Electric field (dBV/m) along the aircraft trajectory.

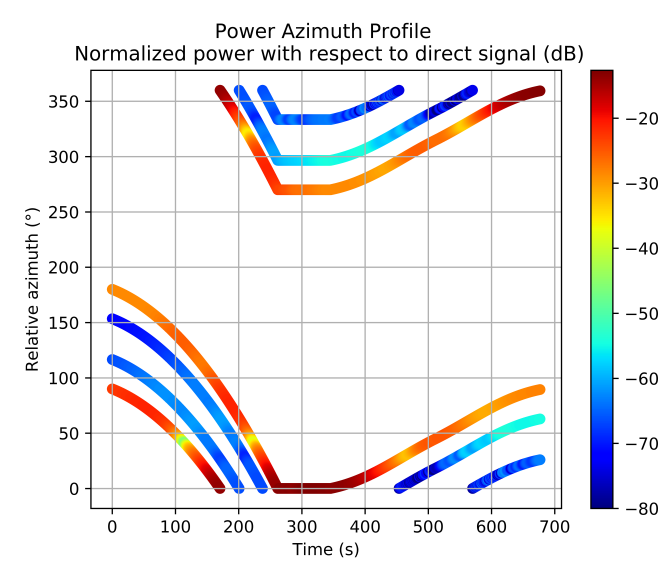

Fig. 4. Power Azimuth Profile of the multipath along the trajectory.

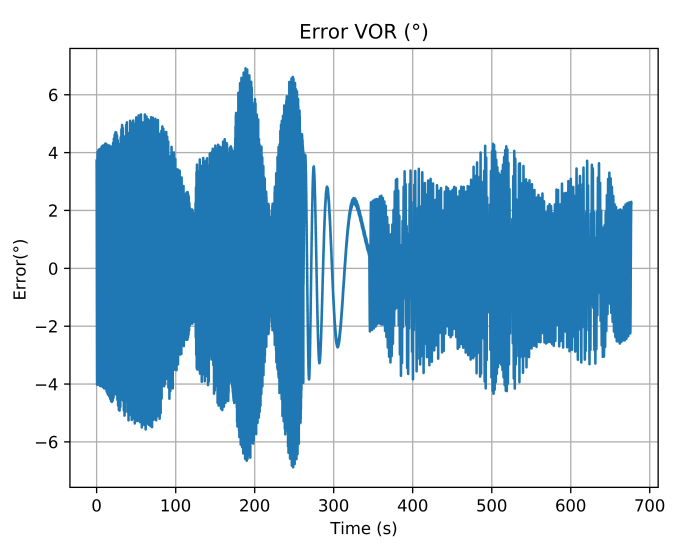

Fig. 5. VOR Error along the trajectory. 


\section{REFERENCES}

[1] C. Morlaas, M. Fares, and B. Souny, "Wind turbine effects on VOR system performance," IEEE Transactions on Aerospace and Electronic Systems, vol. 44, no. 4, pp. 1464-1476, 2008.

[2] F. Fontan, D. Marote, A. Mayo, B. Sanmartin, A. Castro, and J. Navarro, "Assessing multipath induced errors in VOR systems using ray-tracing techniques in combination with detailed terrain databases," in International Flight Inspection Symposium, 2006, pp. 91-96.

[3] D. de la Vega, C. Fernandez, O. Grande, I. Angulo, D. Guerra, Y. Wu, P. Angueira, and J. Ordiales, "Software tool for the analysis of potential impact of wind farms on radiocommunication services," in IEEE International Symposium on Broadband Multimedia Systems and Broadcasting (BMSB), June 2011.

[4] S. Odunaiya and D. Quinet, "Calculations and analysis of signal processing by various navigation receivers architectures," in Digital Avionics Systems Conference, vol. 1, 2004.

[5] L. Claudepierre, R. Douvenot, A. Chabory, and C. Morlaas, "A deterministic VOR error modeling method - Application to wind turbines," IEEE Transactions on Aerospace and Electronic Systems, vol. 53, no. 1, pp. 247-257, 2017.

[6] _ - "A method for computing the VOR multipath error - Comparisons with in-flight measurements," in European Conference on Antennas and Propagation (EuCAP), Davos, Suisse, Apr. 2016. 